\title{
Higher discriminants and the topology of algebraic maps
}

\author{
Luca Migliorini and Vivek Shende
}

\begin{abstract}
Given a map between algebraic varieties, we define loci in the target of the map which we name the "higher discriminants." They are defined in terms of transversality conditions, and, in case of a map between smooth varieties, they can be determined by a tangent space calculation. We prove that the higher discriminants control the variation of cohomology of the fibers in the following two senses: (1) the support of any summand of the pushforward of the intersection cohomology sheaf along a projective map is a component of a higher discriminant, and (2) any component of the characteristic cycle of the proper pushforward of the constant function is a conormal variety to a component of a higher discriminant. As an example, in the last section of the paper, we show that in the case of an algebraic completely integrable system, the stratification by higher discriminants gives exactly the $\delta$-stratification introduced by Ngô.
\end{abstract}

\section{Introduction}

Let $f: X \rightarrow Y$ be a proper family of algebraic varieties. Suppose given a satisfactory understanding of the cohomology of the general fiber and perhaps of some particularly well-behaved special fibers. What can be said about the cohomology of an arbitrary fiber?

Where the map $f$ is smooth, all fibers have the same cohomology. Over $\mathbb{C}$, the theory of Whitney stratifications assures us of the existence of some stratification of $Y$ such that topological properties of the fiber are constant in each stratum [Ver76]. Any question about the cohomology of fibers could be answered by checking at the generic point of every Whitney stratum. Unfortunately, there tend to be a vast number of strata - at least as many as topological types of fibers - which are moreover hard to characterize even in small examples. Checking any fact on each stratum is prohibitive.

Our purpose here is to explain a better strategy. First, we review two points of view on how to think of cohomology in families. For simplicity, in this introduction we focus on complex varieties.

Received 3 April 2016, accepted in final form 4 January 2017.

2010 Mathematics Subject Classification 14D05 (primary), 14F45, 32S60 (secondary).

Keywords: stratification theory, singular support, local Euler obstruction, intersection cohomology.

This journal is (c) Foundation Compositio Mathematica 2018. This article is distributed with Open Access under the terms of the Creative Commons Attribution Non-Commercial License, which permits non-commercial reuse, distribution, and reproduction in any medium, provided that the original work is properly cited. For commercial re-use, please contact the Foundation Compositio Mathematica.

The research of Migliorini was partially supported by projects PRIN 2012 and PRIN 2015 "Spazi di moduli e teoria di Lie". The research of Shende was partially supported by the NSF grant DMS-1406871 and a Sloan fellowship. 


\section{HigheR DISCRIMINANTS OF ALGEBRAIC MAPS}

\subsection{Microlocal geometry}

MacPherson's Chern class morphism from the vector space Con $(X)$ of constructible functions to homology,

$$
\text { c: } \operatorname{Con}(X) \rightarrow H_{*}(X),
$$

commutes with proper pushforwards and is normalized by the condition that for smooth, proper $X$, we have $\mathbf{c}\left(\mathbf{1}_{X}\right)=c(T X) \cap[X]$. Any answer to our question almost necessarily involves coming to terms with it: we want to understand the family of cohomologies of fibers $R f_{*} \mathbb{Q}_{X}$. A simpler question is to understand the family of Euler characteristics of fibers $f_{*} \mathbf{1}_{X}$, and a still simpler question is to understand $\mathbf{c}\left(f_{*} \mathbf{1}_{X}\right)=f_{*} \mathbf{c}\left(\mathbf{1}_{X}\right)$.

We recall the microlocal perspective on these issues [KS90, Ken90]. To a constructible function $\xi$ on a smooth variety $Z$, one can assign the conical Lagrangian $S S(\xi)$ of co-directions in $T^{*} Z$ along which it fails to remain constant; see Definition 2.4. This is called the singular support. Each component can be weighted by the generic amount by which the function changes; the result is a conical Lagrangian cycle in $T^{*} Z$ called the characteristic cycle. This gives an isomorphism between the free abelian groups of constructible functions and conical Lagrangian cycles. The space of conical Lagrangian cycles $\operatorname{Lag}\left(T^{*} Z\right)$ has a geometrically natural basis given by the closures $\overline{T_{S}^{*} Z}$ of conormal bundles to smooth, locally closed subvarieties. So, in our setting $f: X \rightarrow Y$, we expand

$$
C C\left(f_{*} \mathbf{1}_{X}\right)=\sum_{\alpha} n_{\alpha}\left[\overline{T_{V_{\alpha}}^{*} Y}\right] .
$$

To return to constructible functions, we invoke the Brylinski-Dubson-Kashiwara local index formula [BDK81, Gin86]

$$
\begin{aligned}
C C^{-1}: \operatorname{Lag}\left(T^{*} Z\right) & \stackrel{\sim}{\rightarrow} \operatorname{Con}(Z) \\
{\left[\overline{T_{S}^{*} Z}\right] } & \mapsto(-1)^{\operatorname{codim} S} \operatorname{Eu}_{S} .
\end{aligned}
$$

The "Euler obstruction" $\mathrm{Eu}_{S}$ is a constructible function intrinsic to a variety $S$ and taking value 1 at smooth points, originally defined by MacPherson in terms of the Nash transform. By the above index formula, we have

$$
f_{*} \mathbf{1}_{X}=\sum_{\alpha}(-1)^{\operatorname{codim} V_{\alpha}} n_{\alpha} \cdot \operatorname{Eu}_{V_{\alpha}} .
$$

There is a pushforward of conical Lagrangian cycles commuting with the characteristic cycle transformation [Mac74, Ken90, KS90]. From $f: X \rightarrow Y$, one forms $\tilde{f}: T^{*} Y \times_{Y} X=f^{*}\left(T^{*} Y\right) \rightarrow$ $T^{*} Y$ and the differential $d f: T^{*} Y \times_{Y} X \rightarrow T^{*} X$. These give a correspondence

$$
T^{*} X \stackrel{d f}{\longleftarrow} T^{*} Y \times_{Y} X \stackrel{\tilde{f}}{\rightarrow} T^{*} Y .
$$

This correspondence gives the pushforward of Lagrangian cycles, and in particular

$$
S S\left(f_{*} \mathbf{1}\right) \subseteq \tilde{f}\left(d f^{-1}\left(0_{X}\right)\right)
$$

where $0_{X}$ denotes the zero section of $T^{*} X$. Using this formula in practice means figuring out what the components $V_{\alpha}$ of $\tilde{f}\left(d f^{-1}\left(0_{X}\right)\right)$ are. The purpose of the present work is to give a characterization of these components in terms of more readily computable quantities.

\subsection{The decomposition theorem}

The second point of view comes from the decomposition theorem of Beilinson, Bernstein, and Deligne [BBD82]. A special case of this asserts that if $X$ is smooth and $f: X \rightarrow Y$ is projective, 


\section{Migliorini And V. Shende}

then

$$
R f_{*} \mathbb{Q}=\bigoplus_{\alpha} I C\left(U_{\alpha}, \mathcal{L}_{\alpha}\right)\left[n_{\alpha}\right]
$$

In words, $R f_{*} \mathbb{Q}$ splits as a direct sum of shifted simple perverse sheaves. One such simple perverse sheaf, generically on its support $U_{\alpha}$, is just a simple locally constant sheaf $\mathcal{L}_{\alpha}$; the perverse sheaf can be uniquely recovered from $\mathcal{L}_{\alpha}$ by a complicated procedure called intermediate extension. This separates the problem into two steps: first, determine the $U_{\alpha}$ and $\mathcal{L}_{\alpha}$; second, understand the intermediate extension.

The decomposition theorem itself leaves the identity of the $U_{\alpha}$ as a mystery, but some results constraining them are known. If $X$ is nonsingular and the map $f$ is semismall, then the $U_{\alpha}$ which appear are precisely the relevant strata [BM83, dCM02]; see Remark 2.15. For any $f$, if the maximum fiber dimension is $d$, then the $U_{\alpha}$ which appear can be shown by the relative hard Lefschetz theorem to have codimension at most $d$, with strict inequality over the locus where the fibers are irreducible ("Goresky-MacPherson inequality"; see [Ngô10, Théorème 7.3.1]). Finally, there is the recent and celebrated result of Ngô [Ngô10, Ngô09], which applies to the case when $f: X \rightarrow Y$ is a certain sort of relative compactification of a sufficiently well-behaved abelian scheme and implies, for instance, that all the $U_{\alpha}$ which occur are contained in the locus where the fibers $f^{-1}(y)$ are not integral. Finding a general set-up for this result and its generalizations was the main motivation for this paper.

\subsection{Higher discriminants}

We now rephrase our goal: to understand $f_{*} \mathbf{1}_{X}$ and $R f_{*} \mathbb{Q}_{X}$, we want to identify the varieties $V_{\alpha}$ which appear in equation (1.1) and the varieties $U_{\alpha}$ which appear in equation (1.2). To this end, we introduce the higher discriminants. When $Y$ is smooth (a more general formulation appears in Section 2.4), these are given by

$$
\Delta^{i}(f):=\left\{y \in Y \mid \text { no }(i-1) \text {-dimensional subspace of } T_{y} Y \text { is transverse to } f\right\} .
$$

This determines a stratification

$$
Y=\Delta^{0}(f) \supseteq \Delta^{1}(f) \supseteq \Delta^{2}(f) \supseteq \Delta^{3}(f) \supseteq \cdots .
$$

Observe that $\Delta^{1}(f)$ is by definition the locus where the fiber is singular, that is, the usual discriminant. By generic smoothness,

$$
\operatorname{codim} \Delta^{i}(f) \geqslant i
$$

We think about these discriminants in the following way. Moving $\delta \in \Delta(f)$ off the discriminant to $\not \varnothing \notin \Delta^{1}(f)$ changes the fiber topology: $X_{\delta} \nsim X_{\not \varnothing}$. But we can blur our focal point to obscure this feature: we pass to a one-dimensional disc $\mathbb{D} \ni \delta$, chosen generic and small enough to retract $f^{-1}(\mathbb{D})=: X_{\mathbb{D}} \sim X_{\delta}$. A one-dimensional disc cannot be perturbed off the discriminant, and indeed for $\delta$ general in $\Delta^{1}(f)$, a perturbation $\mathbb{D}^{\prime}$ of the thickening $\mathbb{D}$ induces a homeomorphism $X_{\mathbb{D}^{\prime}} \sim X_{\mathbb{D}}$. The higher discriminant $\Delta^{2}(f)$ is the locus which still appears to our blurred vision: where even a general perturbation of a general one-parameter thickening changes the fiber topology. 


\section{HigheR DISCRIMINANTS OF ALGEBRAIC MAPS}

Our main result is that all the $V_{\alpha}$ and $U_{\alpha}$ of Sections 1.1 and 1.2 are irreducible components of higher discriminants.

TheOREm A. If $f: X \rightarrow Y$ is a proper map of smooth algebraic varieties, then any component of the characteristic cycle of $f_{*} \mathbf{1}_{X}$ is the conormal variety to an $i$-codimensional component of $\Delta^{i}(f)$ for some $i$.

TheOREM B. If $f: X \rightarrow Y$ is a projective map of smooth algebraic varieties, then any component of the support of a summand of $R f_{*} \mathbb{Q}_{X}$ is an $i$-codimensional component of $\Delta^{i}(f)$ for some $i$.

We will deduce Theorem A from the following result.

Theorem C. If $f: X \rightarrow Y$ is a proper map of smooth algebraic varieties, then $\tilde{f}\left(d f^{-1}\left(0_{X}\right)\right)$ is the union over all $i$ of the conormals to all the $i$-codimensional components of $\Delta^{i}(f)$.

In fact, in characteristic zero, this implies Theorem B as well: the conormal to the support of any summand of a sheaf is necessarily a component of the singular support (note that here it is essential that we use singular support rather than characteristic cycle, where cancellation may arise), so any such conormal will be a component of $S S\left(f_{*} \mathbb{Q}\right) \subseteq \tilde{f}\left(d f^{-1}\left(0_{X}\right)\right)$.

In characteristic $p$, however, we do not have a good notion of constructible functions or characteristic cycles; see Section 3.

Theorem B, however, can at least be sensibly stated in any characteristic. We give a proof in Section 3 which works generally, under additional assumptions on the codimension of the higher discriminants which are automatic in characteristic zero.

\subsection{Applications}

Theorems A, B, and $\mathrm{C}$ factor the problem of understanding $f_{*} \mathbf{1}$ or $R f_{*} \mathbb{Q}$ into two pieces: first, by computing derivatives, determine the loci $\Delta^{i}(f)$; second, understand their singularities well enough to compute Euler obstructions or intersection cohomology sheaves. Note that the second step no longer depends on the map $f$.

We remark briefly on two ways that such results may be used. One way, following [Ngô10], is that given two maps $f: X \rightarrow B$ and $g: Y \rightarrow B$, one can deduce comparisons of cohomology on all fibers from comparisons of cohomology on sufficiently generic fibers. Our results here imply that "sufficiently generic" means "the general point of each higher discriminant." We have used this method in [MS13, MSV15]; more precisely, our definition here of higher discriminants is distilled from the method we used in [MS13], and our results here are applied in [MSV15].

Of course, doing so requires computing the higher discriminants. This, however, is a tangent space calculation. For example, in Section 4, we describe (following ideas of Dima Arinkin) how the higher discriminants can be computed in the case of an integrable system. This recovers the support theorem of [Ngô10] in this case, but in fact more: it characterizes the microsupport as well.

A different application appears in [ST13], where the cohomology of fibers of a map $f: X \rightarrow B$ is bounded in terms of the cohomology of the general fiber and the singularities of the higher discriminants.

Finally, the paper [dCMM17] contains a detailed study of the supports of the summands considered in Theorem B in the case of a toric map between toric varieties. It would be interesting to see if the supports in this case coincide with the higher discriminants. 


\section{Migliorini And V. Shende}

\section{From singular support to higher discriminants}

In this section, varieties are assumed to be real-analytic manifolds. According to [Ver76], realanalytic stratifications can be refined to Whitney stratifications and provide a good setting for constructible sheaf theory. We will use the word "sheaf" to mean a complex of sheaves of abelian groups and "constructible sheaf" for a complex whose cohomology sheaves are locally constant on each stratum of some real-analytic stratification and have perfect stalks [KS90, Exercise I.30]. We denote by $\mathrm{D}_{c}^{b}(Z)$ the corresponding derived category of constructible sheaves on $Z$. Given a locally closed smooth $V \subseteq Z$, we denote by $T_{V}^{*} Z \subseteq T^{*} Z$ its conormal bundle:

$$
T_{V}^{*} Z=\left\{(z, \phi) \in T^{*} Z \mid z \in V \text { and } T_{z} V \subseteq \operatorname{Ker} \phi\right\} .
$$

\subsection{Review of the singular support of sheaves and transversality}

To a complex of sheaves $\mathcal{F}$ on a real manifold $Z$, one can assign the locus of co-directions in the cotangent bundle along which it fails to remain locally constant. This is its singular support, a conical Lagrangian subvariety of the cotangent bundle; see [KS90, Chapter V] and [VW13, §3] for a clear recollection of the properties of the singular support. A covector $p=(z, \xi) \in T^{*} Z$ does not belong to $S S(\mathcal{F})$ if there exists an open neighborhood $U$ of $p$ in $T^{*} Z$ such that for any $z^{\prime} \in Z$ and any real function $\phi$ defined in a neighborhood of $z^{\prime}$ such that $d \phi_{z^{\prime}} \in U$ and $\phi\left(z^{\prime}\right)=0$, one has

$$
\left(R \Gamma_{\{\phi \geqslant 0\}} \mathcal{F}\right)_{z^{\prime}}=0 .
$$

From its very definition, $S S(\mathcal{F})$ is a conical subset, that is, invariant by the action of $\mathbb{R}_{>0}$ on $T^{*} Z$. The definition of singular support makes sense for an arbitrary bounded complex of sheaves, and by [KS90, Theorem 6.5.4], the singular support $S S(\mathcal{F})$ is an involutive (or co-isotropic) subset of $T^{*} Z$. If $\mathcal{F}$ is constructible, then $S S(\mathcal{F})$ is furthermore Lagrangian [KS90, Theorem 8.4.2].

Singular support transforms well under certain natural operations. Given a map $f: X \rightarrow Y$ of real-analytic manifolds, we write

$$
T^{*} X \stackrel{d f}{\longleftarrow} X \times_{Y} T^{*} Y \stackrel{\tilde{f}}{\rightarrow} T^{*} Y .
$$

We write $f_{\dagger}:=\tilde{f} \circ(d f)^{-1}$ and $f^{\dagger}=(d f) \circ \tilde{f}^{-1}$ for the corresponding maps between subsets of $T^{*} X$ and subsets of $T^{*} Y$.

The convolution $f_{\dagger}$ controls singular supports of proper pushforwards: according to [KS90, Proposition 5.4.4], we have

$$
S S\left(R f_{*} \mathcal{F}\right) \subseteq f_{\dagger} S S(\mathcal{F})
$$

if the restriction of $f$ to the support of $\mathcal{F}$ is proper.

Recall that for a nonsingular variety $Z$, we denote by $0_{Z}=T_{Z}^{*} Z \subset T^{*} Z$ its zero section, clearly a Lagrangian subvariety. Notice the important special case of formula (2.1): if $\mathcal{F}=\mathbb{Q}_{X}$ with $X$ smooth, then $S S\left(\mathbb{Q}_{X}\right)=0_{X}$ and (2.1) becomes

$$
S S\left(R f_{*} \mathbb{Q}_{X}\right) \subseteq f_{\dagger}\left(0_{X}\right)=\left\{(y, \varphi) \in T^{*} Y \mid \exists x \in f^{-1}(y) \text { such that } \varphi \circ d f_{x}=0\right\} .
$$

Definition 2.1. Given a conical subset $A \subseteq T^{*} Y$, we say that $f: X \rightarrow Y$ is transverse to $A$ if

$$
A \cap f_{\dagger}\left(0_{X}\right) \subseteq 0_{Y},
$$

that is, no nonzero covector in $A$ annihilates $d f_{x}\left(T_{x} X\right)$ for $x \in f^{-1}(y)$. We say that a submanifold $U \subseteq Y$ is transverse to $A$ if the inclusion map is transverse to $A$. Finally, we say that a subspace $V \subseteq T_{y} Y$ is transverse to $A$ if no nonzero covector in $A_{y}=A \cap T_{y}^{*} Y$ annihilates $V$. 


\section{HighER DISCRIMINANTS OF ALGEBRAIC MAPS}

Remark 2.2. If $A_{y}$ is a vector subspace of $T_{y} Y^{*}$, hence of the form $A_{y}=U^{\perp}$ with $U \subseteq T_{y} Y$, then no subspace $W \subseteq T_{y} Y$ with $\operatorname{dim} W<\operatorname{dim} A_{y}$ is transverse to $A_{y}$, while there exists an open dense subset of $\left(\operatorname{dim} A_{y}\right)$-dimensional subspaces of $T_{y} Y$ which are transverse to $A_{y}$.

\subsection{Review of constructible functions}

In this section, we work in the complex-analytic set-up. The group of constructible functions $\mathscr{C}(Z)$ on a complex-analytic variety $Z$ is the free abelian group generated by characteristic functions of closed analytic subvarieties. We recall below some relevant facts; for a detailed treatment see, for example, [KS90, §9.7] or [Sch03, § 2.3].

A map $f: X \rightarrow Y$ induces a proper pushforward $f_{!}: \mathscr{C}(X) \rightarrow \mathscr{C}(Y):$ if $W \subseteq X$ is a closed subvariety and $\mathbf{1}_{W}$ is its characteristic function, then $f_{!} \mathbf{1}_{W}(y):=\chi_{c}\left(f^{-1}(y) \cap W\right)$. We have written the compactly supported Euler characteristic to emphasize its additivity, but recall that for algebraic varieties, the compactly supported and usual Euler characteristics agree (see, for example, [Ful93, Note 13] or [Sch03, § 6.0.6]). We employ Viro's integral notation [Vir88]: if $\pi_{Z}: Z \rightarrow$ point is the structure map, then for $\xi \in \mathscr{C}(Z)$ we write $\int_{Z} \xi d \chi:=\left(\pi_{Z}\right) ! \xi$. Explicitly, if $\xi=\sum \xi_{\alpha} \mathbf{1}_{Z_{\alpha}}$, then

$$
\int_{Y} \xi d \chi:=\sum_{\alpha} \xi_{\alpha} \chi_{c}\left(Y_{\alpha}\right) .
$$

Because all the strata are locally contractible, we have the following result.

Lemma 2.3. Fix $\xi \in \mathscr{C}(Z)$. Then for any $z \in Z$, there exists an $\epsilon_{0}>0$ such that, for $0<\epsilon \leqslant \epsilon_{0}$,

$$
\xi(z)=\int_{\|z\|<\epsilon} \xi d \chi
$$

We recall that for a subvariety $V \subseteq Z$, one can define the Euler obstruction $\operatorname{Eu}_{V}(z$ ) (see [Mac74]), a constructible function with support on $V$. It satisfies $\operatorname{Eu}_{V}(z)=1$ if $z$ is a smooth point, and it is constant along strata of a Whitney stratification and preserved by taking products with smooth spaces. There is a hyperplane formula: assuming $\operatorname{dim} V>0$ and taking a local embedding at some $v \in V \subseteq \mathbb{C}^{n}$,

$$
\int_{\mathbb{D}^{n-1}} \operatorname{Eu}_{V} d \chi=\operatorname{Eu}_{V}(v)
$$

for a general disc $\mathbb{D}^{n-1}$ passing near (but not through) $v$; see [BDK81], and also [BLS00, Theorem 3.1] and [Sch02]. The functions $\mathrm{Eu}_{V}$ give a basis for $\mathscr{C}(X)$.

Constructible sheaves have associated constructible functions: with $\mathcal{F} \in \mathrm{D}_{c}^{b}(Z)$, we associate

$$
[\mathcal{F}] \in \mathscr{C}(Z), \quad[\mathcal{F}](z)=\sum(-1)^{i} \operatorname{dim} \mathcal{H}^{i}(\mathcal{F})_{z}
$$

The map $\mathcal{F} \rightarrow[\mathcal{F}]$ factors through the Grothendieck group and is compatible with pushforward: $f_{*}[\mathcal{F}]=\left[\mathrm{R} f_{*} \mathcal{F}\right]$. Clearly, $\mathbf{1}_{W}=\left[\mathbb{Q}_{W}\right]$ for a closed subvariety $W$.

The formalism of nearby and vanishing cycles (see [KS90, $\S 8.6]$ for their definition and the convention for shifts employed here) extends to constructible functions; we recall here enough to fix the notation. Given a regular function $\ell: Z \rightarrow \mathbb{C}$ and $Z_{0}=\ell^{-1}(0)$, we have the distinguished triangle of complexes of sheaves on $Z_{0}$

$$
\left.\Phi_{\ell} \mathcal{F} \rightarrow \mathcal{F}\right|_{Z_{0}} \rightarrow \Psi_{\ell} \mathcal{F} \stackrel{[1]}{\longrightarrow}
$$

The functors $\Phi$ and $\Psi$ descend to operators on constructible functions [Ver81, Propositions 3.4 and 4.1]. If $Z$ is a complex space and $\ell: Z \rightarrow \mathbb{C}$ a holomorphic function, we have the vanishing 


\section{Migliorini And V. Shende}

and nearby operators on constructible functions $\Psi_{l}, \Phi_{l}: \mathscr{C}(Z) \rightarrow \mathscr{C}\left(\ell^{-1}(0)\right)$ as follows: given $\xi \in \mathscr{C}(Z)$, a point $p \in \ell^{-1}(0)$, and $\epsilon \gg \delta$,

$$
\Psi_{\ell} \xi(p):=\int_{B_{\epsilon}(p) \cap \ell^{-1}(\delta)} \xi d \chi, \quad \Phi_{l} \xi(p):=\xi(p)-\Psi_{l} \xi(p) .
$$

If $\mathcal{F}$ is a constructible sheaf, evidently $\Phi_{\ell}[\mathcal{F}]=\left[\Phi_{\ell} \mathcal{F}\right]$ and similarly $\left[\Psi_{\ell} \mathcal{F}\right]=\Psi_{\ell}[\mathcal{F}]$.

We may rewrite the hyperplane formula (2.3) for the Euler obstruction of a subvariety $V \subseteq Z$ as

$\left(\Phi_{\ell} \operatorname{Eu}_{V}\right)(z)=0 \quad$ for $\operatorname{dim} V>0$ and $\ell: Z \rightarrow \mathbb{C}$ a general coordinate function near $z$.

Definition 2.4 (Singular support of a constructible function). For $\xi \in \mathscr{C}(Z)$, a covector $p=$ $\left(z, \lambda_{0}\right) \in T^{*} Z$ does not belong to $S S(\xi)$ if there exists an open neighborhood $U$ of $p$ such that for any $z^{\prime} \in Z$ and any real function $\ell$ defined in a neighborhood of $z^{\prime}$ such that $d \ell_{z^{\prime}} \in U$ and $\ell\left(z^{\prime}\right)=0$, one has $\Phi_{\ell}(\xi)=0$.

Note that the definition is compatible with the map $\mathrm{D}_{c}^{b}(Z) \rightarrow \mathscr{C}(Z)$, in the sense that we have the inclusion $S S([\mathcal{F}]) \subseteq S S(\mathcal{F})$, which may be proper as the example of $\mathbb{Q}_{0} \oplus \mathbb{Q}_{0}[1]$ on $\mathbb{A}^{1}$ shows. If $\xi$ is constructible with respect to a decomposition $\mathcal{S}=\left\{Z_{\alpha}\right\}$ of $Z$, which, after refining, we may suppose to be a Whitney stratification, then $S S(\xi) \subseteq \bigcup T_{Z_{\alpha}}^{*} Z$ and it follows from (2.2), that is, [KS90, Proposition 5.4.4], that we have the inclusions

$$
S S\left(f_{*} \mathbf{1}\right) \subseteq S S\left(R f_{*} \mathbb{Q}\right) \subseteq f_{\dagger}\left(0_{X}\right) .
$$

\subsection{Higher discriminants}

In order to organize the discussion of transversality, we introduce higher discriminants. For this, recall that in Definition 2.1, we introduced the notion of transversality of a vector subspace in $T_{z} Z$ to a conical subset of $T^{*} Z$ :

Definition 2.5. Let $C \subseteq T^{*} Z$ be a conical subvariety. We define

$$
\Delta^{i}(C):=\left\{z \in Z \mid \text { no }(i-1) \text {-dimensional subspace of } T_{z} Z \text { is transverse to } C\right\} .
$$

Clearly, when we work in the complex-analytic category, we refer to the complex dimension in the definition above.

We write $\Delta^{i}(C)_{\text {reg }}$ for the locus where $\Delta^{i}(C)$ is locally a manifold of codimension $i$.

Remark 2.6. If $C^{\prime} \subseteq C$, then $\Delta^{i}\left(C^{\prime}\right) \subseteq \Delta^{i}(C)$ and $\Delta^{i}\left(C^{\prime}\right)_{\text {reg }} \subseteq \Delta^{i}(C)_{\text {reg. }}$.

As we discussed above, by [KS90], the singular support of a constructible sheaf or function is conical Lagrangian. If $\mathcal{F}$ is constructible with respect to the Whitney stratification $\mathcal{S}=\left\{S_{\alpha}\right\}$ of $Z$, then

and similarly for a function.

$$
S S(\mathcal{F}) \subseteq \bigcup T_{S_{\alpha}}^{*} Z
$$

The higher discriminants control the decomposition of a conical Lagrangian into irreducible components. To state the result precisely, recall that in the category of real manifolds, conical only means "invariant under $\mathbb{R}_{\geqslant 0}$ "; we write $\mathbb{R} C$ to take the negative scalars as well. This distinction will be forgotten when we return to complex geometry. 


\section{HigheR DISCRIMINANTS OF ALGEBRAIC MAPS}

Theorem 2.7. If $C \subseteq T^{*} Z$ is a closed conical subanalytic Lagrangian subset, then we have $\operatorname{codim} \Delta^{i}(C) \geqslant i$. Moreover,

$$
\mathbb{R} C=\bigcup_{i} \overline{T_{\Delta^{i}(C)_{\mathrm{reg}}}^{*}}
$$

Proof. Choose a Whitney subanalytic stratification of $Z$ such that $\mathbb{R} C$ is contained in a union of closures of conormal bundles to strata (see [KS90, Proposition 8.3.10]). Observe that the Whitney condition A amounts to the assertion that if $S^{\prime}$ is a stratum inside the closure of $S$, then $\left.\overline{T_{S}^{*} Z}\right|_{S^{\prime}} \subseteq T_{S^{\prime}}^{*} Z$. It follows that $C$ is contained in the union of (not closures of) conormal bundles to strata; increasing $C$ to this union only enlarges $\Delta^{i}(C)$ (by Remark 2.6), so we are free to do this.

To now prove the first claim, we need only consider strata of codimension at most $i$, above which $C$ is the conormal bundle, to which any general $i$-dimensional subspace of the tangent space is transverse.

As for the second statement, the inclusion $T_{\Delta^{i}(C)_{\text {reg }}} Z \subseteq \mathbb{R} C$ is obvious. For the reverse inclusion, observe that if $C \neq \bigcup_{i} \overline{T_{\Delta^{i}(C)_{\text {reg }}} Z}$, then there must be a full-dimensional component $C_{0}$ of $C$ not included in this union. On a dense open subset, we can identify $C_{0}=T_{V}^{*} Z$ for some $V$. But then $V \subseteq \Delta^{\operatorname{codim} V}(C)$; since codim $\Delta^{\operatorname{codim} V}(C)=\operatorname{codim} V$, we have equality along the smooth locus. This gives a contradiction.

Applying this result to a conical Lagrangian of the form $f_{\dagger}\left(0_{Y}\right)$, we immediately have the following result (see Remark 2.2).

Lemma 2.8. Let $f: X \rightarrow Y$ be a map of smooth manifolds. Then $\Delta^{i}\left(f_{\dagger} 0_{X}\right)$ is the locus in $Y$ of points $y \in Y$ such that no $(i-1)$-dimensional subspace of $T_{y} Y$ is transverse to $f$.

Definition 2.9 (Higher discriminants of a map). We write $\Delta^{i}(f):=\Delta^{i}\left(f_{\dagger} 0_{X}\right)$.

Remark 2.10. If $f$ is proper, then $\Delta^{i}(f)$ is closed and, moreover, $\Delta^{>i}(f) \subseteq \Delta^{i}(f)$ by the openness of the transversality condition.

Definition 2.11 (Higher discriminants of constructible sheaves and functions). If $F$ is a constructible function or a complex of constructible sheaves on the nonsingular variety $Z$, we set $\Delta^{i}(F):=\Delta^{i}(S S(F))$.

Translating the definitions of the higher discriminants and the singular support gives the following lemma.

LEMma 2.12. The higher discriminant $\Delta^{i}(F)$ is the locus of $z \in Z$ such that for the general $i$-dimensional local complete intersection $\mathbb{D}^{i} \ni x$ and general linear form $\ell:\left(\mathbb{D}^{i}, z\right) \rightarrow(\mathbb{C}, 0)$, we have $\Phi_{\ell}\left(\left.F\right|_{\mathbb{D}^{i}}\right)(z) \neq 0$.

Theorems A, B, C, whose statements we recall, follow immediately from the discussion above. TheOREM C. If $f: X \rightarrow Y$ is a proper map of smooth algebraic varieties, $f_{\dagger}\left(0_{X}\right)=\tilde{f}\left(d f^{-1}\left(0_{X}\right)\right)$ is the union over all $i$ of the conormals to all the $i$-codimensional components of $\Delta^{i}(f)$.

Proof. This follows from Theorem 2.7 and Lemma 2.8.

Theorem $\mathrm{C}$ and the inclusions (2.6) give Theorem A. 


\section{Migliorini And V. Shende}

Theorem A. If $f: X \rightarrow Y$ is a proper map of algebraic varieties, then any component of the characteristic cycle of $f_{*} \mathbf{1}_{X}$ is the conormal variety to an $i$-codimensional component of $\Delta^{i}(f)$ for some $i$.

Finally, the decomposition theorem and the observation that if $\mathcal{F}$ is a summand of $R f_{*} \mathbb{Q}_{X}$, then the conormal bundle to the support of $\mathcal{F}$ is a component of $S S\left(R f_{*} \mathbb{Q}_{X}\right)$ give Theorem B.

Theorem B. If $f: X \rightarrow Y$ is a projective map of algebraic varieties, then any component of the support of a summand of $R f_{*} \mathbb{Q}_{X}$ is an $i$-codimensional component of $\Delta^{i}(f)$ for some $i$.

We end this section with two examples from complex geometry clarifying the notion of higher discriminant.

Example 2.13 (Higher discriminants and stratification by the rank of the differential). The locus $\Delta^{i}(f)$ may be larger than the image of the locus in $X$ where $d f$ has cokernel of dimension at least $i$. Indeed, let $f$ be the blow-up of a point $p$ on a smooth surface: the image of $d f$ has dimension one at every point of the exceptional divisor. Nevertheless, $p \in \Delta^{2}(f)$ : no one-dimensional disc in the base is transverse to all these tangent space images simultaneously, and indeed the inverse image of every one-dimensional disc $\mathbb{D}$ through $p$ will be given by the union of the proper transform of $\mathbb{D}$ and the exceptional divisor, which is singular.

On the other hand, it may well happen that a stratum for the map is not a higher discriminant.

Example 2.14 (The versal deformation of the cusp curve). Let $f: X \subseteq \mathbb{A}^{2} \times \mathbb{P}^{2} \rightarrow \mathbb{A}^{2}=Y$ be the family of projective curves

$$
\left\{(a, b,[X, Y, Z]) \in \mathbb{A}^{2} \times \mathbb{P}^{2} \mid Z Y^{2}-X^{3}-a X Z^{2}-b Z^{3}=0\right\} .
$$

If $\Delta \subseteq \mathbb{A}^{2}$ is defined by

$$
\left\{(a, b) \in \mathbb{A}^{2} \mid 4 a^{3}+27 b^{2}=0\right\},
$$

a stratification for the map is given by

$$
\left\{(0,0) \subseteq \Delta \subseteq \mathbb{A}^{2}\right\}
$$

Nevertheless, just as for the other points of $\Delta$, the inverse image of a one-dimensional complex disc through the origin has nonsingular total space. Therefore, $\Delta^{2}(f)=\emptyset$ and $\Delta^{1}(f)=\Delta$. Notice that in this case $\Delta^{1}(f)$ does not support any summand of $R f_{*} \mathbb{Q}_{X}$.

Remark 2.15. Let $f: X \rightarrow Y$ be a semismall map, with $X$ nonsingular, and assume $f(X)=Y$. Define $Y_{i}=\left\{y \in Y\right.$ such that $\left.\operatorname{dim} f^{-1}(y)=i\right\}$. A component $Y_{\alpha}$ of $Y_{i}$ supports a summand of $R f_{*} \mathbb{Q}_{X}$ if and only if $\operatorname{dim} Y_{\alpha}=\operatorname{dim} Y-2 i$ (these are the relevant strata; see [BM83, dCM02]). It follows from Theorem 3.4 that in this case $\overline{Y_{\alpha}} \subseteq \Delta^{2 i}(f)$. This means that there is no $(2 i-1)$-dimensional disc through the general point of $Y_{\alpha}$ with nonsingular inverse image of the right codimension. For example, if a point $y$ is a zero-dimensional relevant stratum, namely $\operatorname{dim} f^{-1}(y)=\frac{1}{2} \operatorname{dim} X$, then there is no Cartier divisor through $x$ whose inverse image is a nonsingular Cartier divisor in $X$. Notice that the consideration of the dimension of the fiber would give a much cruder estimate, namely that there is no $i$-dimensional disc with nonsingular inverse image of the right codimension.

\subsection{Remarks on the singular case}

The above ideas make immediate sense when $Y$ is singular: one simply chooses locally an embedding of $Y$ into a smooth manifold. Below, we comment on what can be done when $X$ is singular. 


\section{HigheR DISCRIMINANTS OF ALGEBRAIC MAPS}

We omit the proofs, both because they are standard and because, although it is possible to set up the theory, we do not know in practice how to compute the higher discriminants.

Fix an embedding of $X$ in a smooth manifold $\tilde{X}$. Let $\mathcal{S}$ be the canonical Whitney stratification of $(\widetilde{X}, X)$ constructed in [Tei82, Chapter VI, $\S 3$ ]. Let $T_{\mathcal{S}}^{*} X$ be the union of all conormals to strata in $Y$; by the Whitney conditions, this is closed.

Given a map $f: X \rightarrow Y$, fix an extension $\widetilde{f}: \widetilde{X} \rightarrow Y$. Then we can take $f_{\dagger} 0_{X}:=\widetilde{f}_{\dagger}\left(0_{X}\right)$, which gives a conical subvariety of $Y$. We can again define $\Delta^{i}(f):=\Delta^{i}\left(f_{\dagger} 0_{X}\right)$.

Lemma 2.8 has the following variant: $\Delta^{i}(f)$ is the locus of $y \in Y$ such that there is no $(i-1)$ germ of a complete intersection $\mathbb{D}^{i-1}$ passing through $y$ for which the inclusion $f^{-1}\left(\mathbb{D}^{i-1}\right):=$ $\left(X \times_{Y} \mathbb{D}^{i-1}\right) \hookrightarrow X$ is normally nonsingular; that is, it admits a neighborhood in $X$ homeomorphic to a bundle over itself and $\operatorname{codim}\left(\mathbb{D}^{i-1}, Y\right)=\operatorname{codim}\left(f^{-1}\left(\mathbb{D}^{i-1}\right), X\right)$.

With these definitions, Theorem $\mathrm{C}$ again holds. In Theorem $\mathrm{A}$, it is natural to replace $\mathbb{Q}_{X}$ with the intersection cohomology sheaf with respect to some perversity. The result holds in all of these cases because, with the above definitions, using [GM83, $\S 5.4 .1$ ], we have $S S\left(I C_{X}\right) \subseteq T_{X}^{*} X$, hence $S S\left(f_{*} I C_{X}\right) \subseteq f_{\dagger}\left(S S\left(I C_{X}\right)\right) \subseteq f_{\dagger} T_{X}^{*} X$, hence $\Delta^{i}\left(S S\left(f_{*} I C_{X}\right)\right) \subseteq \Delta^{i}(f)$. Theorem B will hold with $\mathbb{Q}_{X}$ replaced by the middle-perverse intersection cohomology sheaf.

\subsection{Theorem A: reformulations and applications}

We now give some corollaries of Theorem 2.7. Throughout this section, we work with complex varieties. For $X$ smooth and $S \subseteq X$, we write $\overline{T_{S}^{*} X}$ for the closure of the conormal bundle of the smooth locus of $S$. We denote by $C C(\phi)$ the characteristic cycle of the constructible function $\phi$. Its definition in terms of vanishing cycles, discussed in [Bry86, § I.1], gives the following result.

Corollary 2.16. Let $X$ be a complex variety, and let $\xi$ be a constructible function on $X$. Let $\left\{\Delta^{i, \alpha}\right\}_{\alpha}$ be the $i$-codimensional components of $\Delta^{i}(\xi)$. For $x_{i, \alpha} \in \Delta^{i, \alpha}$ a general point, $X \supset$ $\mathbb{D}^{i} \ni x_{i, \alpha}$ a general $i$-dimensional disc, and $l: \mathbb{D}^{i} \rightarrow \mathbb{C}$ a general linear form, define $\xi^{i, \alpha}=$ $\Phi_{l}\left(\left.\xi\right|_{\mathbb{D}^{i}}\right)\left(x_{i, \alpha}\right)$. Then

$$
C C(\xi)=\sum(-1)^{i} \xi^{i, \alpha} \overline{T_{\Delta^{i, \alpha}}^{*} X}
$$

By the Brylinski-Dubson-Kashiwara index theorem [BDK81, Gin86], we have $C C\left(\mathrm{Eu}_{S}\right)=$ $(-1)^{\operatorname{codim} S}\left[\overline{T_{S}^{*} Y}\right]$, giving the following corollary.

Corollary 2.17. With the hypotheses and notation of Corollary 2.16,

$$
\xi=\sum_{i, \alpha} \xi^{i, \alpha} \mathrm{Eu}_{\overline{\Delta^{i, \alpha}}}
$$

Finally, assume that $f: X \rightarrow Y$ is a proper map of complex varieties. Since $S S\left(f_{*} \mathbf{1}_{X}\right) \subseteq$ $f_{\dagger} S S\left(\mathbf{1}_{X}\right)=f_{\dagger} T_{X}^{*} X[\mathrm{KS} 90]$, the following holds.

Corollary 2.18. Let $f: X \rightarrow Y$ be a proper map of complex varieties. Let $\left\{\Delta^{i, \alpha}\right\}_{i, \alpha}$ be the codimension $i$ components of $\Delta^{i}(f)$. Then

$$
\begin{aligned}
C C\left(f_{*} \mathbf{1}_{X}\right) & =\sum_{i, \alpha}(-1)^{i} \xi^{i, \alpha} \overline{T_{\Delta^{i, \alpha}}^{*} Y} \\
f_{*} \mathbf{1}_{Y} & =\sum \xi^{i, \alpha} \mathrm{Eu}_{\overline{\Delta^{i, \alpha}}} .
\end{aligned}
$$




\section{Migliorini And V. Shende}

\section{Theorem B in characteristic $p$}

In positive characteristic $p$, finding a good notion of constructible functions or characteristic cycles turns out to be extremely subtle. The main problem is wild ramification: for instance, the map

$$
\text { as: } \mathbb{A}^{1} \stackrel{x \mapsto x^{p}-x}{\longrightarrow} \mathbb{A}^{1}
$$

is a nontrivial cover whose source is a space with Euler characteristic one; composing with the pushforward to a point gives a counterexample to the possibility that the naive pushforward of constructible functions is functorial. There is some recent important progress on this topic [AS07, Sai09, Sai15].

Similarly, easy examples with inseparable morphisms show that the estimate (1.3) on the codimension of the higher discriminants may not hold. In the recent paper [Bei16], Beilinson defines a notion of singular support for étale constructible sheaves on an algebraic variety $X$ over an arbitrary field, as a closed subset of $T^{*} X$, and proves that every irreducible component has dimension $\operatorname{dim} X$. However, the examples given in [Bei16] show that this is not as well behaved as in characteristic zero; in particular, the singular support is not necessarily a union of conormals to subvarieties of $X$. Therefore, even assuming that the estimate (1.3) holds, the methods used in the previous sections cannot be extended. Here we give another proof of Theorem $\mathrm{B}$, for the direct image of the constant sheaf on a nonsingular variety, also valid over positive-characteristic fields, under the supplementary hypothesis that the codimension estimate (1.3) holds.

Throughout, we employ the formalism of perverse sheaves [BBD82]. For $Z$ an algebraic variety and $K \in \mathrm{D}_{c}^{b}(Z)$ a constructible complex, we denote by $\mathscr{H}^{i}(K)$ its $i$ th perverse cohomology sheaf. We adopt the usual shift convention: if $Z$ is smooth, then $\mathbb{Q}_{Z}[\operatorname{dim} Z]\left(\operatorname{or} \mathbb{Q}_{l, Z}[\operatorname{dim} Z]\right.$ in the étale setting) is perverse. We work in some setting where a formalism of weights is available, either with $\ell$-adic sheaves over a finite field $\mathbb{F}_{q}$ or with mixed Hodge modules over $\mathbb{C}$. We say that $K \in \mathrm{D}_{c}^{b}(Z)$ is pure semisimple of weight $k$ if it is isomorphic to the direct sum of its perverse cohomology sheaves and for every $i$, the sheaf $p_{\mathscr{H}}^{i}(K)$ is a semisimple pure perverse sheaf of weight $i+k$.

We reformulate the definition of higher discriminants of a map in this context.

Terminology: Let $Y$ be a nonsingular variety. Given $y \in Y$, by "a $k$-dimensional disc $\mathbb{D}^{k} \hookrightarrow Y$ through $y$," we mean a germ of a nonsingular $k$-dimensional subvariety passing through $y$.

Definition 3.1. Let $f: X \rightarrow Y$ be a proper map of nonsingular algebraic varieties. A $k$ dimensional disc $\mathbb{D}^{k} \hookrightarrow Y$ through $y \in Y$ is transverse to $f$ if $f^{-1}\left(\mathbb{D}^{k}\right)$ is nonsingular along $f^{-1}(y)$ and $\operatorname{codim}\left(f^{-1}\left(\mathbb{D}^{k}\right), X\right)=\operatorname{codim}\left(\mathbb{D}^{k}, Y\right)$.

Definition 3.2. Let $f: X \rightarrow Y$ be a proper map of nonsingular algebraic varieties. Then

$$
\Delta^{i}(f)=\left\{y \in Y \mid \text { there is no } \mathbb{D}^{i-1} \hookrightarrow Y \text { through } y \text { transverse to } f\right\} .
$$

Remark 3.3. The definition generalizes immediately to the case of $Y$ locally embeddable into a smooth variety; all the results hold mutatis mutandis for such $Y$.

\subsection{Supports are discriminants}

The decomposition theorem of [BBD82] asserts that if $f: X \rightarrow Y$ is proper and $X$ is nonsingular, then $R f_{*} \mathbb{Q}$ (or $R f_{*} \mathbb{Q}_{\ell}$ ) is pure semisimple. In other words, there are nonsingular locally closed subvarieties $V_{i} \subseteq Y$ carrying semisimple local systems $L_{i}$ such that $R f_{*} \mathbb{Q}=\bigoplus \operatorname{IC}\left(V_{i}, L_{i}\right)\left[d_{i}\right]$ for appropriate $d_{i} \in \mathbb{Z}$. 


\section{HigheR DISCRIMINANTS OF ALGEBRAIC MAPS}

Theorem 3.4. Let $f: X \rightarrow Y$ be a projective map between algebraic varieties, with $X$ nonsingular. Let $\mathcal{G}$ be a summand of $R f_{*} \mathbb{Q}_{\ell}$, and let $k$ be the codimension of supp $\mathcal{G}$. Then

$$
\operatorname{supp} \mathcal{G} \subseteq \Delta^{k}(f) .
$$

In particular, if, for every $k$, we have codim $\Delta^{k}(f) \geqslant k$, then every summand of $R f_{*} \mathbb{Q}_{\ell}$ is supported on the closure of a $k$-codimensional component of $\Delta^{k}(f)$.

Often, even in characteristic $p$, it is possible to just directly make the tangent space calculation determining the $\Delta^{k}(f)$ and directly show that $\Delta^{k}(f)$ has codimension at least $k$. For an application, see [MSV15].

We turn to giving the proof of Theorem 3.4. First, we develop some preliminary notions.

Definition 3.5. We write $\mathfrak{S}(Z) \subseteq \mathrm{D}_{c}^{b}(Z)$ for the set of pure semisimple complexes $K$ which are moreover symmetric in the sense that $p^{p} \mathcal{H}^{i}(K) \simeq \mathfrak{H}^{-i}(K)(-i)$ for every $i \in \mathbb{Z}$.

Remark 3.6. (i) If $K, K[r] \in \mathfrak{S}(Z)$, then either $K=0$ or $r=0$.

(ii) If $K=K_{1} \oplus K_{2}$ and two of the three complexes are in $\mathfrak{S}(Z)$, then so is the third.

(iii) If $K \in \mathfrak{S}(Z)$ and $K_{\Lambda}$ is the sum of the summands of $K$ with support exactly equal to $\Lambda \subseteq Z$, then $K_{\Lambda} \in \mathfrak{S}(Z)$.

Lemma 3.7 ([BBD82, Corollaire 4.1.12]). If $P$ is a simple perverse sheaf on $Z$ and $i: D \hookrightarrow Z$ is a Cartier divisor, then either

(i) $\operatorname{supp} P \subsetneq D$ and $i^{*} P[-1]$ is perverse, or

(ii) $\operatorname{supp} P \subseteq D$ and $i^{*} P$ is perverse.

Corollary 3.8. Let $Z$ be a variety, and let $i: D \hookrightarrow Z$ denote the closed immersion of a Cartier divisor. If $K \in \mathfrak{S}(Z)$ and $i^{*} K[-1] \in \mathfrak{S}(D)$, then $D$ does not contain the support of any summand of $K$.

Proof. Write $K=K^{\prime} \oplus i_{*} K^{\prime \prime}$, where $i_{*} K^{\prime \prime}$ is the direct sum of all the summands whose support is contained in $D$. By Remark 3.6(ii), we have $K^{\prime}, i_{*} K^{\prime \prime} \in \mathfrak{S}(Z)$. By hypothesis, $i^{*} K$ and hence $i^{*} K^{\prime}$ and $i^{*} i_{*} K^{\prime \prime}=K^{\prime \prime}$ have semisimple perverse cohomology sheaves. By Lemma 3.7, (up to Tate twists)

$$
{ }^{p} \mathscr{H}^{k}\left(i^{*} K^{\prime}[-1]\right)=\left(i^{*} \mathscr{H}^{k}\left(K^{\prime}\right)\right)[-1] \simeq\left(i^{*} \mathscr{H}^{-k}\left(K^{\prime}\right)\right)[-1]={ }^{p} \mathscr{H}^{-k}\left(i^{*} K^{\prime}[-1]\right)
$$

and so $i^{*} K^{\prime}[-1] \in \mathfrak{S}(D)$. Thus, since $i^{*} K[-1]=i^{*} K^{\prime}[-1] \oplus K^{\prime \prime}[-1] \in \mathfrak{S}(D)$, we have $K^{\prime \prime}[-1] \in$ $\mathfrak{S}(D)$. On the other hand, obviously $K^{\prime \prime} \in \mathfrak{S}(D)$, which, by Remark 3.6(i), gives a contradiction unless $K^{\prime \prime}=0$.

Proof of Theorem 3.4. As $f$ is projective and, by the smoothness of $X$, the sheaf $\mathcal{F}:=\mathbb{Q}_{\ell}[\operatorname{dim} X]$ is pure and perverse, it follows from the decomposition theorem and the relative hard Lefschetz theorem that $R f_{*} \mathcal{F} \in \mathfrak{S}(X)$.

We first show that the zero-dimensional supports of the summands of $R f_{*} \mathcal{F}$ are contained in $\Delta^{\operatorname{dim} Y}(f)$. Indeed, if $y \notin \Delta^{\operatorname{dim} Y}(f, \mathcal{F})$, then by definition there exists a divisor $i: D \rightarrow Y$ containing $y$ such that $\widetilde{D}:=f^{-1}(D)$ is nonsingular in a neighborhood of $f^{-1}(y)$. Thus $i^{*} \mathcal{F}[-1]$ is pure perverse; hence, by the decomposition theorem, $R\left(\left.f\right|_{\widetilde{D}}\right)_{*}\left(i^{*} \mathcal{F}[-1]\right) \in \mathfrak{S}(D)$.

By proper base change,

$$
R\left(\left.f\right|_{\tilde{D}}\right)_{*}\left(i^{*} \mathcal{F}[-1]\right)=i^{*} R f_{*} \mathcal{F}[-1]
$$




\section{Migliorini And V. Shende}

in a neighborhood of $y$. Therefore $i^{*} R f_{*} \mathcal{F}[-1] \in \mathfrak{S}(D)$ and, by Corollary 3.8, there is no summand of $R f_{*} \mathcal{F}$ supported on $y$.

In the general case, let $Z$ be the support of a simple summand $\mathcal{G}$ of $R f_{*} \mathcal{F}$, and denote its codimension by $k$. We show that $z \in \Delta^{k}(f)$. If not, then, there is a $(k-1)$-dimensional disc $\mathbb{D}^{k-1}$ through $z$ transverse to $f$. Since transversality is an open condition, there is a $k$-dimensional disc $\mathbb{D}^{k}$ through $z$ containing $\mathbb{D}^{k-1}$ and transverse to $f$. Consider the restriction $f_{\mid}: f^{-1}\left(\mathbb{D}^{k}\right) \rightarrow \mathbb{D}^{k}$. By proper base change, there is a nonzero summand of $\left.R f_{\mid *} \mathcal{F}[k-\operatorname{dim} Y]\right|_{\mathbb{D}^{k}}$ supported at $z$, but also a codimension one disc through $z$ transverse to $f_{\mid}$, and we find a contradiction from what we proved in the first step.

Remark 3.9. In [MS13, $\S 5]$, we proved (but did not state) a weaker form of Theorem 3.4.

Remark 3.10. One might hope for a more precise form of Theorem 3.4, holding for the direct image of a pure perverse sheaf $\mathcal{F}$ on $Y$, in which the role of the higher discriminants $\Delta^{i}(f)$ is played by the "discriminants of pure perversity" $\Delta_{p}^{i}(f, \mathcal{F})$, defined as follows: Say that a disc $\mathbb{D}^{i-1} \rightarrow Y$ through $y \in Y$ is transverse if $\left.\mathcal{F}\right|_{f^{-1}\left(\mathbb{D}^{i-1}\right)}\left[-\operatorname{codim}\left(\mathbb{D}^{i-1}, Y\right)\right]$ is pure perverse. Then define $\Delta_{p}^{i}(f, \mathcal{F})$ to be the locus of $y \in Y$ through which there is no $(i-1)$-dimensional transverse $\operatorname{disc} \mathbb{D}^{i-1} \rightarrow Y$. Unfortunately, it is not clear whether these discriminants have the required properties to make the argument of Theorem 3.4 work. Clearly, $\Delta_{p}^{i}(f, \mathbb{Q}) \subseteq \Delta^{i}(f)$, and it is easy to find examples in which inclusion is strict, for example with finite maps, but, on the other hand, the determination of these discriminants looks quite hard.

\section{Higher discriminants of algebraic completely integrable systems}

In this section, we show how one can determine the higher discriminants of an algebraic completely integrable system. We are grateful to Dima Arinkin for explaining to us the following argument [AF16, Ngô09], which sheds light on the link between the " $\delta$-regularity" assumption and the support theorem in [Ngô10]. We make the following assumptions (see [DM96, AF16]):

(i) The scheme $X$ is a complex nonsingular quasi-projective variety, endowed with an algebraic symplectic form $\Omega$.

(ii) The map $h: X \rightarrow Y$ is a flat projective map with connected and reduced fibers to a nonsingular $d$-dimensional affine variety $Y$ with $2 \operatorname{dim} Y=\operatorname{dim} X$. The restriction of $\omega$ to the smooth locus of any fiber is zero.

(iii) There exists a commutative group scheme $G \rightarrow Y$ acting on $X \rightarrow Y$, with $\operatorname{dim} G=\operatorname{dim} X$, making $h$ into a completely integrable system: For every point $x \in X$, let $d h_{x}: T_{x} X \rightarrow T_{h(x)} Y$ be the differential and

$$
{ }^{t} d h: T_{h(x)}^{*} Y \rightarrow T_{x}^{*} X \stackrel{\Omega}{\cong} T_{x} X
$$

its adjoint. Then we have an isomorphism $\operatorname{Lie}\left(G_{h(x)}\right) \cong T_{h(x)}^{*} Y$, and the derivative of the action is given by the linear map

$$
\operatorname{Lie}\left(G_{h(x)}\right) \cong T_{h(x)}^{*} Y \stackrel{t^{t} d h}{\longrightarrow} T_{x}^{*} X \stackrel{\Omega}{\cong} T_{x} X
$$

above.

(iv) On a dense open set $Y_{o} \subseteq Y$, the group scheme $G_{o}=G \times_{Y} Y_{o} \rightarrow Y_{o}$ is an abelian scheme.

(v) For every $x \in X$, the stabilizer $S(x)<G_{h(x)}$ is affine. 


\section{HigheR DISCRIMINANTS OF ALGEBRAIC MAPS}

Remark 4.1. Let $\mathscr{M}(n, d)$ be the moduli space of stable Higgs bundles $(E, \Phi)$ of rank $n$ and degree $d$ on a genus $g$ projective curve, where $d$ and $n$ are assumed coprime and $g \geqslant 2$. Let $h: \mathscr{M}(n, d) \rightarrow \mathbb{A}$ be the Hitchin map. We recall that the points of $\mathbb{A}$ parameterize certain Cartier divisors contained in the total space of the canonical bundle $K_{C}$ of $C$, the spectral curves. Let $\mathbb{A}_{\text {red }} \subset \mathbb{A}$ be the open set corresponding to reduced spectral curves and $\mathscr{M}(n, d)_{\text {red }}:=h^{-1}\left(\mathbb{A}_{\text {red }}\right)$. The hypotheses above are satisfied by the restriction of the Hitchin map

$$
h_{\mid}: \mathscr{M}(n, d)_{\text {red }} \rightarrow \mathbb{A}_{\text {red }} .
$$

A detailed study of the supports of the Hitchin map, using the methods developed in the present paper, is pursued in a forthcoming paper by M. A. de Cataldo, J. Heinloth, and L. Migliorini.

Recall that, by the theorem of Chevalley, for every $y \in Y$, the neutral component $G_{y}^{o}$ of the commutative algebraic group $G_{y}$ is an extension

$$
1 \rightarrow H_{y} \rightarrow G_{y}^{o} \rightarrow A_{y} \rightarrow 1
$$

of an abelian variety $A_{y}$ by a connected affine algebraic group $H_{y}$, whence an upper-semicontinuous function

$$
\delta: Y \rightarrow \mathbb{N}, \quad \delta(y)=\operatorname{dim} H_{y} .
$$

In particular, for every $y \in Y$, we have the subspace $\operatorname{Lie}\left(H_{y}\right) \subseteq \operatorname{Lie}\left(G_{y}\right) \cong T_{y}^{*} Y$. By semicontinuity, the fourth assumption amounts to asking that $\delta^{-1}(0) \neq \emptyset$. The last assumption on the stabilizers is not particularly restrictive, in view of the following.

Lemma 4.2. Suppose that a connected algebraic group $G$ acts on a connected variety $Z$. Then either the stabilizers of all points of $Z$ are affine or the stabilizers of all points of $Z$ fail to be affine.

Proof. We may assume $Z$ irreducible. Suppose that there is a point $x \in Z$ whose stabilizer $S(x)<G$ is not affine. The stabilizer acts on the local ring $\mathcal{O}_{Z, x}$; since $S(x)$ is not a linear group, this representation must have a kernel $H(x)$. Since $H(x)$ acts trivially on the local ring $\mathcal{O}_{Z, x}$, it acts trivially on the entire $Z$, and we are left to show that $H(x)$ is not affine.

Clearly, the quotient $S(x) / H(x)$ is affine, having a faithful linear representation. Hence, the kernel $H(x)$ cannot be affine, otherwise $S(x)$ would be an extension of an affine group by an affine group, and therefore affine itself.

Notice also that because of the assumptions made on the dimension and the connectedness of the fibers of $h$, for $y \in Y_{o}$ the group scheme $G_{y}$ acts transitively on $h^{-1}(y)$.

Proposition 4.3. We have $\Delta^{i}(h)=\{y \in Y \mid \delta(y) \geqslant i\}$.

Proof. For $x \in X$, let $y=h(x)$, and let $S_{x} \subseteq G_{y}$ denote the neutral connected component of its stabilizer. Its Lie algebra satisfies $\operatorname{Lie}\left(S_{x}\right) \subseteq \operatorname{Lie}\left(G_{y}\right)=T_{y}^{*} Y$. From the definition of the Hamiltonian action, it follows that

$$
d h\left(T_{x} X\right)=\operatorname{Lie}\left(S_{x}\right)^{\perp} \subseteq T_{y} Y
$$

is the orthogonal complement of this subspace $\operatorname{Lie}\left(S_{x}\right) \subseteq T_{y}^{*} Y$. By hypothesis, $S_{x}$ is affine, hence contained in the maximal affine $H_{y}$. This implies that the images $d h\left(T_{x} X\right)$ for $x \in h^{-1}(y)$ all contain Lie $\left(H_{y}\right)^{\perp}$. By the Borel fixed point theorem [Spr98, Theorem 6.2.6], the Lie algebra $\operatorname{Lie}\left(H_{y}\right)$ is the stabilizer of some $x \in h^{-1}(y)$, and the statement follows from the fact that the codimension of this smallest subspace is exactly $\delta(y)$. 


\section{Migliorini And V. Shende}

From this proposition, one gets a fairly workable criterion for proving support theorems in the line of those in [Ngô10, CL12, CL16]; see the forthcoming paper [MSV15], where compactified Jacobian fibrations associated with family of planar curves are considered in detail.

\section{ACKNOWLEDGEMENTS}

We thank Paolo Aluffi, Daniel Lowengrub, Ngô Bau Chau, Filippo Viviani, Jacob Tsimerman, Melanie Wood, and Geordie Williamson for helpful discussions, and especially Dima Arinkin for the characterization of higher discriminants of integrable systems and Mark A. de Cataldo for giving us many valuable suggestions and pointing out several inaccuracies in the earlier versions of this paper.

\section{REFERENCES}

AF16 D. Arinkin and R. Fedorov, Partial Fourier-Mukai transform for integrable systems with applications to Hitchin fibration, Duke Math. J. 165 (2016), no. 15, 2991-3042; https: //doi.org/10.1215/00127094-3645223.

AS07 A. Abbes and T. Saito, The characteristic class and ramification of an l-adic étale sheaf, Invent. Math. 168 (2007), no. 3, 567-612; https://doi.org/10.1007/s00222-007-0040-7.

BBD82 A. A. Beillinson, J. Bernstein, and P. Deligne, Faisceaux pervers, Astérisque 100 (1982), 5-171.

BDK81 J.-L. Brylinski, A.S. Dubson, and M. Kashiwara, Formule de l'indice pour modules holonomes et obstruction d'Euler locale, C. R. Acad. Sci. Paris Sér. I Math. 293 (1981), no. 12, 573-576.

Bei16 A. Beilinson, Constructible sheaves are holonomic, Selecta Math. (N.S.) 22 (2016), no. 4, 17971819; https://doi.org/10.1007/s00029-016-0260-z.

BLS00 J.-P. Brasselet, Lê Dũng Tráng, and J. Seade, Euler obstruction and indices of vector fields, Topology 39 (2000), no. 6, 1193-1208; https://doi.org/10.1016/S0040-9383(99)00009-9.

BM83 W. Borho and R. MacPherson, Partial resolutions of nilpotent varieties, Astérisque 101 (1983), $23-74$.

Bry86 J.-L. Brylinski, Transformations canoniques, dualité projective, théorie de Lefschetz, transformations de Fourier et sommes trigonométriques, Astérisque 140-141 (1986), 3-134.

CL12 P.-H. Chaudouard and G. Laumon, Le lemme fondamental pondéré. II. Énoncés cohomologiques, Ann. of Math. 176 (2012), no. 3, 1647-1781; https://doi.org/10.4007/annals. 2012.176.3.6.

CL16 Un théorème du support pour la fibration de Hitchin, Ann. Inst. Fourier (Grenoble) 66 (2016), no. 2, 711-727; https://doi.org/10.5802/aif.3023.

dCM02 M. de Cataldo and L. Migliorini, The hard Lefschetz theorem and the topology of semismall maps, Ann. Sci. École Norm. Sup. (4) 35 (2002), no. 5, 759-772; https://doi.org/10.1016/ S0012-9593(02) 01108-4.

dCMM17 M. de Cataldo, L. Migliorini, and M. Mustaţă, The combinatorics and topology of proper toric maps, J. reine angew. Math., published online on 1 March 2016, https://doi.org/10.1515/ crelle-2015-0104, to appear in print.

DM96 R. Donagi and E. Markman, Spectral covers, algebraically completely integrable, Hamiltonian systems, and moduli of bundles, Integrable Systems and Quantum Groups (Montecatini Terme, 1993), Lecture Notes in Math., vol. 1620 (Springer, Berlin, 1996), 1-119; https://doi.org/ 10.1007/BFb0094792.

Ful93 W. Fulton, Introduction to toric varieties, Ann. of Math. Stud., vol. 131 (Princeton Univ. Press, Princeton, NJ, 1993); https://doi.org/10.1515/9781400882526. 


\section{HigheR DISCRIMINANTS OF ALGEBRAIC MAPS}

Gin86 V. Ginsburg, Characteristic varieties and vanishing cycles, Invent. Math. 84 (1986), no. 2, 327-402; https://doi.org/10.1007/BF01388811.

GM83 M. Goresky and R. MacPherson, Intersection homology. II, Invent. Math. 72 (1983), no. 1, 77-129; https://doi.org/10.1007/BF01389130.

Ken90 G. Kennedy, MacPherson's Chern classes of singular algebraic varieties, Comm. Algebra 18 (1990), no. 9, 2821-2839; https://doi.org/10.1080/00927879008824054.

KS90 M. Kashiwara and P. Schapira, Sheaves on manifolds, Grundlehren math. Wiss., vol. 292 (Springer-Verlag, Berlin, 1990); https://doi.org/10.1007/978-3-662-02661-8.

Mac74 R. D. MacPherson, Chern classes for singular algebraic varieties, Ann. of Math. 100 (1974), 423-432; https://doi.org/10.2307/1971080.

MS13 L. Migliorini and V. Shende, A support theorem for Hilbert schemes of planar curves, J. Eur. Math. Soc. 15 (2013), no. 6, 2353-2367; https://doi.org/10.4171/JEMS/423.

MSV15 L. Migliorini, V. Shende, and F. Viviani, A support theorem for hilbert schemes of planar curves, II, 2015, arXiv:1508.07602.

Ngô09 B. C. Ngô, Decomposition theorem and abelian fibration, 2009, Notes from a talk given in Bonn in a conference in honor of M. Rapoport, available at http://www. math.uchicago.edu/ ngo/ support.pdf.

Ngô10_ Le lemme fondamental pour les algèbres de Lie, Publ. Math. Inst. Hautes Études Sci. (2010), no. 111, 1-169; https://doi.org/10.1007/s10240-010-0026-7.

Sai09 T. Saito, Wild ramification and the characteristic cycle of an l-adic sheaf, J. Inst. Math. Jussieu 8 (2009), no. 4, 769-829; https://doi.org/10.1017/S1474748008000364.

Sai15 Characteristic cycle and the Euler number of a constructible sheaf on a surface, J. Math. Sci. Univ. Tokyo 22 (2015), no. 1, 387-441; http://www.ms.u-tokyo.ac.jp/ journal/abstract_e/jms220113_e.html.

Sch02 J. Schürmann, A short proof of a formula of Brasselet, Lê and Seade for the Euler obstruction, 2002, arXiv:math.AG/0201316.

Sch03 Topology of singular spaces and constructible sheaves, Math. Inst. Polish Acad. Sci., Math. Monogr. (N.S.), vol. 63 (Birkhäuser Verlag, Basel, 2003); https://doi.org/10.1007/ 978-3-0348-8061-9.

Spr98 T. A. Springer, Linear algebraic groups, 2nd ed., Progr. Math., vol. 9 (Birkhäuser Boston, Inc., Boston, MA, 1998); https://doi.org/10.1007/978-0-8176-4840-4.

ST13 V. Shende and J. Tsimerman, Equidistribution on the space of rank two vector bundles over the projective line, 2013, arXiv:1307.8237.

Tei82 B. Teissier, Variétés polaires. II. Multiplicités polaires, sections planes, et conditions de Whitney, Algebraic Geometry (La Rábida, 1981), Lecture Notes in Math., vol. 961 (Springer, Berlin, 1982), 314-491; https://doi.org/10.1007/BFb0071291.

Ver76 J.-L. Verdier, Stratifications de Whitney et théorème de Bertini-Sard, Invent. Math. 36 (1976), 295-312; https://doi.org/10.1007/BF01390015.

Ver81_ Spécialisation des classes de Chern, Astérisque 82 (1981), 149-159.

Vir88 O. Ya. Viro, Some integral calculus based on Euler characteristic, Topology and Geometry Rohlin Seminar, Lecture Notes in Math., vol. 1346 (Springer, Berlin, 1988), 127-138; https: //doi.org/10.1007/BFb0082775.

VW13 K. Vilonen and G. Williamson, Characteristic cycles and decomposition numbers, Math. Res. Lett. 20 (2013), no. 2, 359-366; https://doi.org/10.4310/MRL.2013.v20.n2.a11. 


\section{Migliorini And V. Shende}

Luca Migliorini luca.migliorini@unibo.it

Dipartimento di Matematica, Università di Bologna, Piazza di Porta S. Donato 5, 40126 Bologna, Italy

Vivek Shende vivek@math.berkeley.edu

Department of Mathematics, University of California, Berkeley, 970 Evans Hall, Berkeley CA 94720, USA 